\title{
Bounds on Amplification Factors, Element-Node and Node-Node Equivalence for Square Finite Elements in Kinematic Shallow Water Equations
}

Jagadeesh Anmala ( $\sim$ jagadeesh@hyderabad.bits-pilani.ac.in )

Birla Institute of Technology and Science - Hyderabad Campus https://orcid.org/0000-0003-1571-8640 Rabi H Mohtar

American University of Beirut

\section{Research Article}

Keywords: amplification factors, eigen-solutions, nodal solutions, kinematic wave.

Posted Date: December 29th, 2021

DOl: https://doi.org/10.21203/rs.3.rs-776348/v1

License: (c) (1) This work is licensed under a Creative Commons Attribution 4.0 International License.

Read Full License 


\section{Abstract}

The upper and lower bounds of amplification factors of lumped finite element schemes are compared with nodal (integer or half-integer multiple of) eigen-value solutions of consistent finite element scheme at element and node levels of error analysis. The closeness or proximity between bounds on solutions of amplification factors and eigen-solutions reveals that the two methods, consistent and lumped finite element schemes are equivalent. The element error solutions of lumped mass matrix assumption and consistent nodal solution denotes the element-node error equivalence and the nodal solutions of all of the finite element schemes denote the node-node error equivalence for square finite elements in kinematic wave shallow water equations. The comparison plots of lumped and consistent finite element schemes are presented in this paper for illustration.

\subsection{Introduction}

Lumped finite element schemes are developed as alternate modeling schemes of a consistent mass matrix approach to save CPU time and improve upon convergence and stability for nonlinear modeling problems. The addition of all of the column wise-coefficients to the diagonal in each row introduces positive definiteness into the capacitance matrix. It reduces the value of maximum eigen-values in the mass-lumping approach. This increases the allowable time-step and reduces the spurious oscillations at which critical time-step occurs. The effects of mass lumping for kinematic wave shallow water equations are explored by Jaber and Mohtar (2002a, 2002b, 2003) using linear finite element shape functions for two-dimensional problems. The mass-lumping approach increases the time-step size by a factor of two compared to the consistent mass matrix approach to obtain solutions with the same accuracy for kinematic wave shallow water equations. Armentano and Duran (2002) discuss the masslumping for second-order elliptic eigen-value problems. According to their findings, the eigen-values obtained using the mass-lumping approach are below the ones obtained using the continuous approach or exact integration method except for the singular case. Botella (2003) discusses the effects of masslumping and the associated loss in numerical accuracy when the approach is used for compressible flow simulations using Navier-Stokes equation solvers. The objectives of the current study are to: (i) compare lower and upper bound nodal solutions of amplification factors of lumped scheme with consistent eigenvalue nodal solution, (ii) obtain Courant numbers using coefficient method for lumped scheme and by eigen-value analysis for consistent scheme.

\subsection{Methodology}

\subsection{Fourier-von Neumann Analysis of Kinematic Wave Shallow Water Finite Element Schemes}

The continuity equation of linearized shallow water system is given by $\frac{\partial h}{\partial t}+u \frac{\partial h}{\partial x}+v \frac{\partial h}{\partial y}=0$, where $u=\alpha_{x} \beta h^{\beta-1}, v=\alpha_{y} \beta h^{\beta-1}, \alpha_{x}=\sqrt{S_{0 x}} / n_{m}, \alpha_{y}=\sqrt{S_{0 y}} / n_{m}, \beta=5 / 3, S_{0 x}$ and $S_{0 y}$ are the 
element slopes in $x$ and $y$ directions, $u$ and $v$ are the $x$ and $y$ components of velocity, $h$ is the flow depth and $n_{m}$ is the Manning's roughness coefficient (Anmala and Mohtar, 2011). Assuming the finite element solution by $\bar{h}$, we obtain the error as $\epsilon(x, y, t)=h(x, y, t)-\bar{h}(x, y, t)$. Introducing the interpolation functions defined by $\bar{h}=\sum_{j=1}^{N} H_{j}(t) N_{j}(x, y)$ and the residual error is minimized as follows: $\int_{0}^{1} \int_{0}^{1}\left[\bar{h}_{t}+u \bar{h}_{x}+v \bar{h}_{y}\right] N_{j}(x, y) d x d y=0$. Upon simplification, the finite element system can be written as $A H^{\prime}+B_{x} H+B_{y} H+f=0$. The matrices $\mathrm{A}, \mathrm{B}_{\mathrm{x}}, \mathrm{B}_{\mathrm{y}}$ are given by

$$
\begin{gathered}
A=\int_{\Omega_{e}} N^{T} N d \Omega_{e}=\frac{a b}{36}\left[\begin{array}{cccc}
4 & 2 & 1 & 2 \\
2 & 4 & 2 & 1 \\
1 & 2 & 4 & 2 \\
2 & 1 & 2 & 4
\end{array}\right] \\
B_{x}=\int_{\Omega_{e}} N^{T} \frac{\partial N}{\partial x} d \Omega_{e}=\frac{b}{12}\left[\begin{array}{llll}
-2 & 2 & 1 & -1 \\
-2 & 2 & 1 & -1 \\
-1 & 1 & 2 & -2 \\
-1 & 1 & 2 & -2
\end{array}\right] \\
\text { and } B_{y}=\int_{\Omega_{e}} N^{T} \frac{\partial N}{\partial y} d \Omega_{e}=\frac{a}{12}\left[\begin{array}{llll}
-2 & -1 & 1 & 2 \\
-1 & -2 & 1 & 1 \\
-1 & -2 & 2 & 1 \\
-2 & -1 & 2 & 2
\end{array}\right]
\end{gathered}
$$

where $a=\Delta x$ and $b=\Delta y$ for consistent method using the bilinear reactangular interpolation functions $N_{i}=\left(1-\frac{x}{a}\right)\left(1-\frac{y}{b}\right), N_{j}=\left(\frac{x}{a}\right)\left(1-\frac{y}{b}\right), N_{k}=\left(\frac{x}{a}\right)\left(\frac{y}{b}\right), N_{m}=\left(\frac{y}{b}\right)\left(1-\frac{x}{a}\right)$. The element matrix $A$ for the lumped finite element scheme is given by

$A=\frac{a b}{4}\left[\begin{array}{llll}1 & 0 & 0 & 0 \\ 0 & 1 & 0 & 0 \\ 0 & 0 & 1 & 0 \\ 0 & 0 & 0 & 1\end{array}\right]$. Following a similar analysis for error equations represented by $\frac{\partial \epsilon}{\partial t}+u \frac{\partial \epsilon}{\partial x}+v \frac{\partial \epsilon}{\partial y}=0$, we obtain the error element and error gradient matrices. Approximating the error by Fourier series given by $\int_{\Omega_{e}} \epsilon(x, y, t) d \Omega_{e}=\sum_{n=-\infty}^{n=+\infty} \sum_{m=-\infty}^{m=+\infty} e^{i\left(\omega_{m}+\omega_{n}\right) t} e^{i\left(\sigma_{m} x+\sigma_{n} y\right)}$, where $\omega_{m}$ and $\omega_{n}$ are temporal wave numbers and $\sigma_{m}$ and $\sigma_{n}$ are spatial wave numbers in $\mathrm{x}$ and y directions. This series can be approximated using individual error components such as $\epsilon_{i j}^{n}(x, y, t)=e^{a t} e^{i\left(\sigma_{x} \Delta x+\sigma_{y} \Delta y\right)}$, and $\epsilon_{i j}^{n-1}(x, y, t)=e^{-a \Delta t} \epsilon_{i j}^{n}(x, y, t)$. The error equation can be solved for the amplification factor using the temporal wave number $a$. The specifics of stability analysis can be found from Press et al. (1992). 


\subsection{Theory: Lumped vs. Consistent Finite element schemes}

Lumped mass matrix approach gives lower estimates of maximum eigen-values than distributed mass matrix or consistent mass matrix approach. Therefore, the lumped mass matrix would allow a larger timestep or critical time-step than the consistent mass matrix approach. In other words, the stability of a numerical scheme is assured with a larger time-step using mass lumping of capacitance matrix (Anmala and Mohtar, 2011). The issues of convergence and stability of lumped and distributed mass matrix approaches for linear and nonlinear problems are discussed by Wood (1990). The reduction of eigenvalues for first and second-order scalar equations could be seen from distributed mass matrix modeling approach to lumped mass matrix modeling approach. In the same context, the effects of mass lumping for first and second-order problems, computation of eigen-values using Newmark and $\theta$-method are detailed by Wood (1990).

The lower and upper bounds determined using the coefficient method for consistent and lumped element and node solutions are compared with integer multiples of eigen-solutions of consistent approximation. The closeness between the bounding solutions for all schemes with eigen-solutions of consistent approximation shows that the square finite element behaves essentially like a node at a larger scale. Node-node comparisons of amplification factors imply the accuracy of consistent finite element schemes and the alternate lumped finite element schemes. The solutions of amplification factors imply that the linearization of mass matrix coefficients in capacitance matrix and introduction of positive definiteness into the capacitance matrix do not alter the schemes significantly and only helps in obtaining better convergence rate.

\subsection{Bounds on Amplification factors}

The lower and upper bounds on amplification factors using the coefficient method are discussed in Anmala and Mohtar (2011). The lumped mass matrix assumption gives only one bounding estimate on Courant number using the coefficient method for all of the methods: explicit, semi-implicit, and implicit. The eigenvalue analysis of the system of finite element equations of shallow water for all the schemes (explicit, semi-implicit, implicit) and methods (consistent, lumped, upwind) could be obtained from that of Anmala and Mohtar (2015).

\subsection{Results And Discussion}

The results of element-node, node-node error amplification factors are compared in this section for illustration purposes for both the methods: consistent and lumped.

\subsection{Explicit Schemes}

\subsubsection{Explicit element and Consistent node}


Element level error equations are identical for consistent, lumped, and upwind finite element schemes. The amplification factors for the element level error equation and an integer multiple of consistent nodal solution are compared in Fig. 1.

\subsubsection{Explicit node and Consistent node}

The only one bound on amplification factor is obtained using lumped finite element scheme. The amplification factors of the explicit, lumped scheme are compared with that of an integer multiple of consistent nodal solution shown in Fig. 2. The closeness between the solutions implies that the bound obtained using the coefficient method for lumped scheme is essentially an eigen-solution of consistent nodal approximation. The only bounding result of explicit lumped finite element scheme can be obtained from Table 1 and Table 2 (reproduced here as Table 1 and Table 2) of Anmala and Mohtar (2011). Table 1 (reproduced here as Table 3) of Anmala and Mohtar (2015) shows that the eigenvalue of finite element system of the explicit, consistent scheme is 0.35 and 0.70 is an integer multiple eigenvalue. The proximity of both the results is evident in Fig. 2 . 
Table 1. Courant number limit with each scheme $(\Delta x \neq \Delta y, \mu \neq \eta)$.

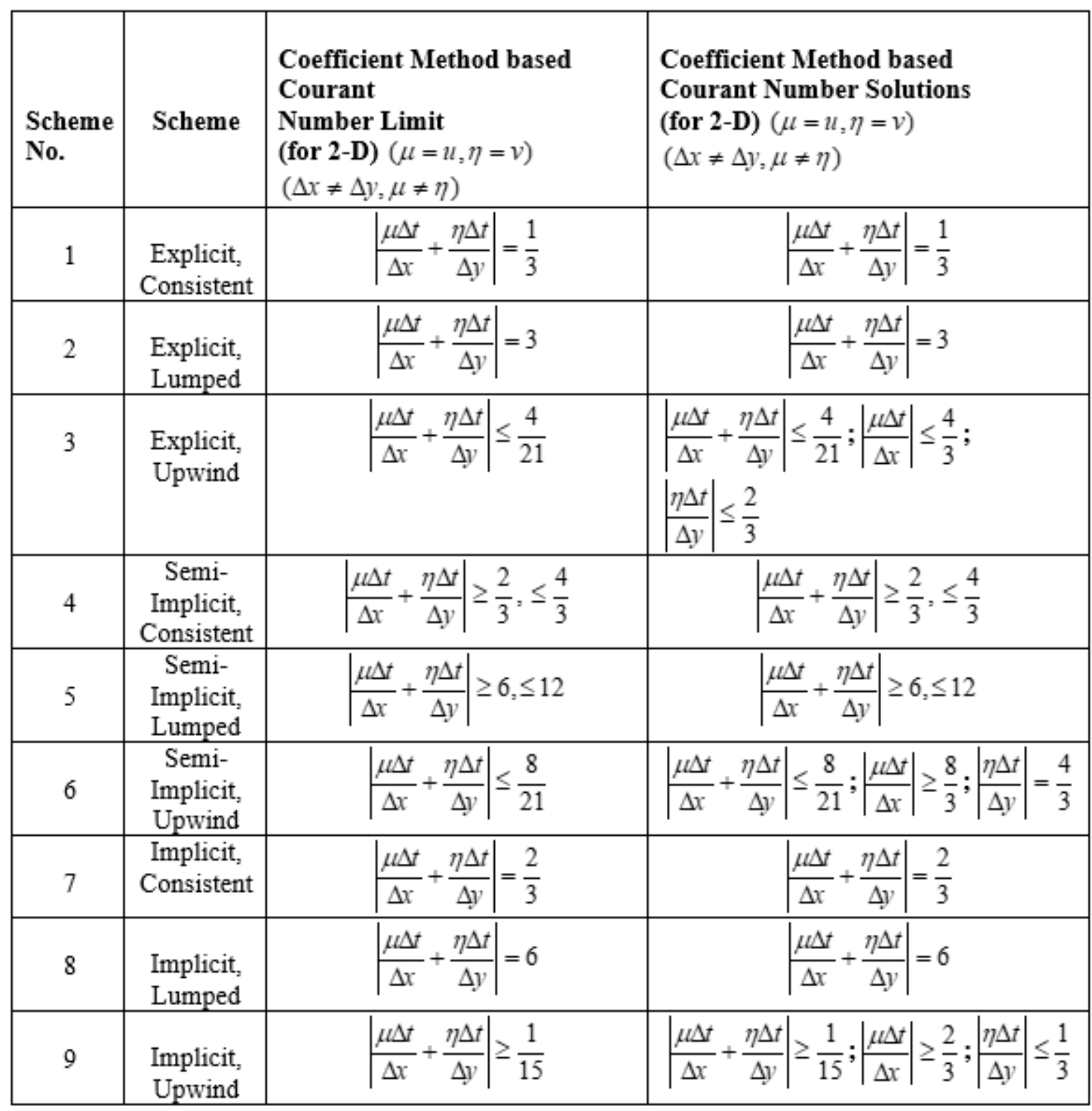


Table 2. Courant number limit for all of the schemes $(\Delta x=\Delta y, \mu=\eta)$.

\begin{tabular}{|c|c|c|c|c|c|}
\hline $\begin{array}{l}\text { Scheme } \\
\text { No. }\end{array}$ & Scheme & $\begin{array}{l}\text { Coefficient } \\
\text { Method based } \\
\text { Courant } \\
\text { Number Limit } \\
\text { (for 2-D, from } \\
\text { column on the } \\
\text { r.h.s) } \\
(\mu=u, \eta=v)\end{array}$ & $\begin{array}{l}\text { Coefficient } \\
\text { Method based } \\
\text { Courant } \\
\text { Number Limit } \\
(\Delta x=\Delta y, \mu=\eta) \\
\left|\frac{\mu \Delta t}{\Delta x}\right| \text { or }\left|\frac{\eta \Delta t}{\Delta y}\right|\end{array}$ & $\begin{array}{l}\text { Upper bound } \\
\text { using } \\
\text { Coefficient } \\
\text { Method } \\
(\Delta x=\Delta y, \mu=\eta) \\
\left|\frac{\mu \Delta t}{\Delta x}\right| \text { or }\left|\frac{\eta \Delta t}{\Delta y}\right|\end{array}$ & $\begin{array}{l}\text { Eigen-value } \\
\text { based time- } \\
\text { step }\end{array}$ \\
\hline 1 & $\begin{array}{c}\text { Explicit, } \\
\text { Consistent }\end{array}$ & $\left|\frac{\mu \Delta t}{\Delta x}+\frac{\eta \Delta t}{\Delta y}\right| \leq \frac{1}{3}$ & $\left|\frac{\mu \Delta t}{\Delta x}\right| \leq \frac{1}{6}$ & $2 / 3$ & $\frac{\mu \Delta t}{\Delta x} \leq \frac{5}{2 \sqrt{51}}$ \\
\hline 2 & $\begin{array}{l}\text { Explicit, } \\
\text { Lumped }\end{array}$ & $\left|\frac{\mu \Delta t}{\Delta x}+\frac{\eta \Delta t}{\Delta y}\right| \leq \frac{3}{2}$ & $\left|\frac{\mu \Delta t}{\Delta x}\right| \leq \frac{3}{4}$ & $3 / 4$ & $\frac{\mu \Delta t}{\Delta x} \leq \frac{5}{4}$ \\
\hline 3 & $\begin{array}{l}\text { Explicit, } \\
\text { Upwind }\end{array}$ & $\left|\frac{\mu \Delta t}{\Delta x}+\frac{\eta \Delta t}{\Delta y}\right| \leq \frac{4}{21}$ & $\left|\frac{\mu \Delta t}{\Delta x}\right| \leq \frac{2}{21}$ & $4 / 3$ & $\frac{\mu \Delta t}{\Delta x} \leq 0.0888$ \\
\hline 4 & $\begin{array}{c}\text { Semi- } \\
\text { Implicit, } \\
\text { Consistent } \\
\end{array}$ & $\left|\frac{\mu \Delta t}{\Delta x}+\frac{\eta \Delta t}{\Delta y}\right| \leq \frac{2}{3}$ & $\left|\frac{\mu \Delta t}{\Delta x}\right| \leq \frac{1}{3}$ & $4 / 3$ & $\frac{\mu \Delta t}{\Delta x} \leq \frac{5}{4 \sqrt{51}}$ \\
\hline 5 & $\begin{array}{c}\text { Semi- } \\
\text { Implicit, } \\
\text { Lumped } \\
\end{array}$ & $\left|\frac{\mu \Delta t}{\Delta x}+\frac{\eta \Delta t}{\Delta y}\right| \leq 3$ & $\left|\frac{\mu \Delta t}{\Delta x}\right| \leq \frac{3}{2}$ & $3 / 2$ & $\frac{\mu \Delta t}{\Delta x} \leq \frac{5}{8}$ \\
\hline 6 & $\begin{array}{c}\text { Semi- } \\
\text { Implicit, } \\
\text { Upwind }\end{array}$ & $\left|\frac{\mu \Delta t}{\Delta x}+\frac{\eta \Delta t}{\Delta y}\right| \leq \frac{8}{21}$ & $\left|\frac{\mu \Delta t}{\Delta x}\right| \leq \frac{4}{21}$ & $8 / 3$ & $\frac{\mu \Delta t}{\Delta x} \leq 0.0444$ \\
\hline 7 & $\begin{array}{l}\text { Implicit, } \\
\text { Consistent }\end{array}$ & $\left|\frac{\mu \Delta t}{\Delta x}+\frac{\eta \Delta t}{\Delta y}\right| \geq \frac{1}{3}$ & $\left|\frac{\mu \Delta t}{\Delta x}\right| \geq \frac{1}{6}$ & $2 / 3$ & $\frac{\mu \Delta t}{\Delta x} \geq \frac{5}{2 \sqrt{51}}$ \\
\hline 8 & $\begin{array}{l}\text { Implicit, } \\
\text { Lumped }\end{array}$ & $\left|\frac{\mu \Delta t}{\Delta x}+\frac{\eta \Delta t}{\Delta y}\right| \geq \frac{3}{2}$ & $\left|\frac{\mu \Delta t}{\Delta x}\right| \geq \frac{3}{4}$ & $3 / 4$ & $\frac{\mu \Delta t}{\Delta x} \geq \frac{5}{4}$ \\
\hline 9 & $\begin{array}{l}\text { Implicit, } \\
\text { Upwind }\end{array}$ & $\left|\frac{\mu \Delta t}{\Delta x}+\frac{\eta \Delta t}{\Delta y}\right| \geq \frac{4}{3}$ & $\left|\frac{\mu \Delta t}{\Delta x}\right| \geq \frac{2}{3}$ & $4 / 3$ & $\frac{\mu \Delta t}{\Delta x} \geq 0.0888$ \\
\hline
\end{tabular}


Table 3

Eigen-values for Finite Element (FE) Schemes.

\begin{tabular}{|llll|}
\hline System Number & Scheme and Method & $\begin{array}{l}\text { FE System } \\
\text { Eigen-value }\end{array}$ & Number of modes \\
\hline 1 & Explicit, Consistent & 0.35 & 3 \\
\hline 2 & Explicit, Lumped & 1.25 & 1 \\
\hline 3 & Explicit, Upwind & 0.0888 & 12 \\
\hline 4 & Semi-Implicit, Consistent & 0.175 & 6 \\
\hline 5 & Semi-Implicit, Lumped & 0.625 & 2 \\
\hline 6 & Semi-Implicit, Upwind & 0.0444 & 24 \\
\hline 7 & Implicit, Consistent & 0.35 & 3 \\
\hline 9 & Implicit, Lumped & 1.25 & 1 \\
\hline
\end{tabular}

\subsection{Semi-Implicit Schemes}

\subsubsection{Semi-Implicit element and Consistent node}

We obtain identical error equations for all of the methods using the semi-implicit scheme. The amplification factors of semi-implicit element solution are compared with that of consistent nodal solution in Fig. 3.

\subsubsection{Semi-Implicit node and Consistent node}

The lumped, semi-implicit, and consistent nodal solutions are compared in Fig. 4. The proximity between the amplification factors implies that the bound obtained using the coefficient method for the semiimplicit, lumped scheme is essentially an eigen-solution of consistent nodal approximation. The only bounding solution of the semi-implicit, lumped scheme can be obtained from Table 1 and Table 2 (reproduced here as Table 1 and Table 2) of Anmala and Mohtar (2011). The semi-implicit, consistent finite element system's eigenvalue of 0.175 can be obtained from Table 1 (reproduced here as Table 3 ) of Anmala and Mohtar (2015). The Courant number of 1.4 is an integer multiple of the eigenvalue of 0.175. The closeness of both the amplification factors against the wavenumbers is due to the closeness between the bounding result and multiplicity of eigenmodes. 


\subsection{Implicit Schemes}

\subsubsection{Implicit element and Consistent node}

Similar error equations are obtained for consistent, upwind, and lumped implicit schemes. The element level amplification factors are compared with implicit, consistent nodal solution and shown in Fig. 5 .

\subsubsection{Implicit node and Consistent node}

The lumped, implicit nodal solutions are compared with that of the consistent nodal solution in Fig. 6 . The closeness between all of the amplification factors reveals that the bound obtained using the coefficient method for the implicit, lumped scheme is essentially an eigen-solution of consistent nodal approximation. The only bounding solution of implicit lumped finite element scheme can be seen from Table 1 and Table 2 (reproduced here as Table 1 and Table 2) of Anmala and Mohtar (2011). The fundamental eigenvalue of the implicit, consistent finite element scheme can be obtained from Table 1 (reproduced here as Table 3) of Anmala and Mohtar (2015). As in explicit and semi-implicit schemes, the bounding result of the lumped finite element scheme is very close to that of integer multiple of implicit, consistent nodal finite element system.

\section{Conclusion}

1. The proximity between the bounds on solutions of amplification factors and eigen-solutions reveals that all of the three schemes are essentially equivalent.

2. The mass-lumping approach reduces maximum eigen-values and increases the allowable time-step for numerical stability. However, when the lumped element and lumped nodal solutions are compared with consistent, nodal solutions of amplification factors, we obtain a very close agreement between the error solutions.

3. The results of the upwind scheme also behave in the same fashion as that of lumped finite element scheme when compared using the consistent, nodal solution and are not presented in this paper for reasons of brevity.

4. The equivalence of proximal-bounds determined using the coefficient method and eigen-value solution essentially imply the element-node and node-node error equivalence in Galerkin-framework for kinematic wave shallow water equations using square finite elements.

\section{Declarations}

\section{Data Availability Statement}

No data were generated or used during the study. 


\section{Acknowledgement}

A USGS grant supported this work through the Indiana Water Resources Center and Department of Science and Technology (DST, Govt. of India) grant (SR/S3/MERC-0001/2011) of the project titled "Fourier Analysis and Development of Two-Dimensional Finite Element Schemes for Shallow Water Equations and Transport related Systems".

\section{Ethical Approval}

This study does not contain any studies with human participants or animals performed by any of the authors.

\section{Funding Details}

This study was supported by a grant from Indiana Water Resources Center, Indiana, USA and DST, Govt. of India (Department of Science and Technology, grant no. SR/S3/MERC-0001/2011).

\section{Conflict of interest}

The authors declare that they have no known competing financial interests or personal relationships that could have appeared to influence the work reported in this paper.

\section{Informed Consent}

Informed consent was obtained from all individual participants included in the study.

\section{Authorship Contributions}

JA and RHM conceived this research. JA and RHM participated in the analysis. JA wrote the original draft. JA and RHM participated in the revisions of the draft. All authors read and approved the final manuscript.

\section{References}

1. Anmala J, Mohtar RH (2011) Fourier Stability Analysis of Two-Dimensional Finite Element Schemes for Shallow Water Equations, International Journal of Computational Fluid Dynamics, 25(02): 75-94. DOI:10.1080/10618562.2011.560572

2. Anmala J, Mohtar RH (2015) Cauchy solution of the Kinematic Wave Shallow Water Equations using Square Grid Finite Element Method, ASCE, Journal of Hydrologic Engineering, 20(12), 04015042, DOI: 10.1061/(ASCE)HE.1943-5584.0001241.

3. Armentano MG, Duran RG (2003) Mass-Lumping or not Mass-Lumping for eigenvalue problems, Numerical methods for partial differential equations, 19: 653-664. 
4. Botella O (2003) A high-order mass-lumping procedure for B-spline collocation method with application to incompressible flow simulations, International Journal for Numerical Methods in Fluids, 41: 1295-1318.

5. Jaber FH, Mohtar RH (2002a) Dynamic time step for the one-dimensional overland flow kinematic wave solution, J Hydrol Eng. ASCE; 7(1):3-11.

6. Jaber FH, Mohtar RH (2002b) Stability and accuracy of finite element schemes for the onedimensional kinematic wave solution, Adv Water Resour, 25:427-38.

7. Jaber FH, Mohtar RH (2003) Stability and accuracy of two-dimensional kinematic wave overland flow modeling, Adv Water Resour, 26:1189-98.

8. Press WH, Flannery BP, Teukolsky SA, Vetterling WT (1992) Numerical Recipes in C: The Art of Scientific Computing, $2^{\text {nd }}$ Edition, Cambridge University Press.

9. Wood WL (1990) Practical Time-stepping schemes, Oxford University Press.

\section{Figures}

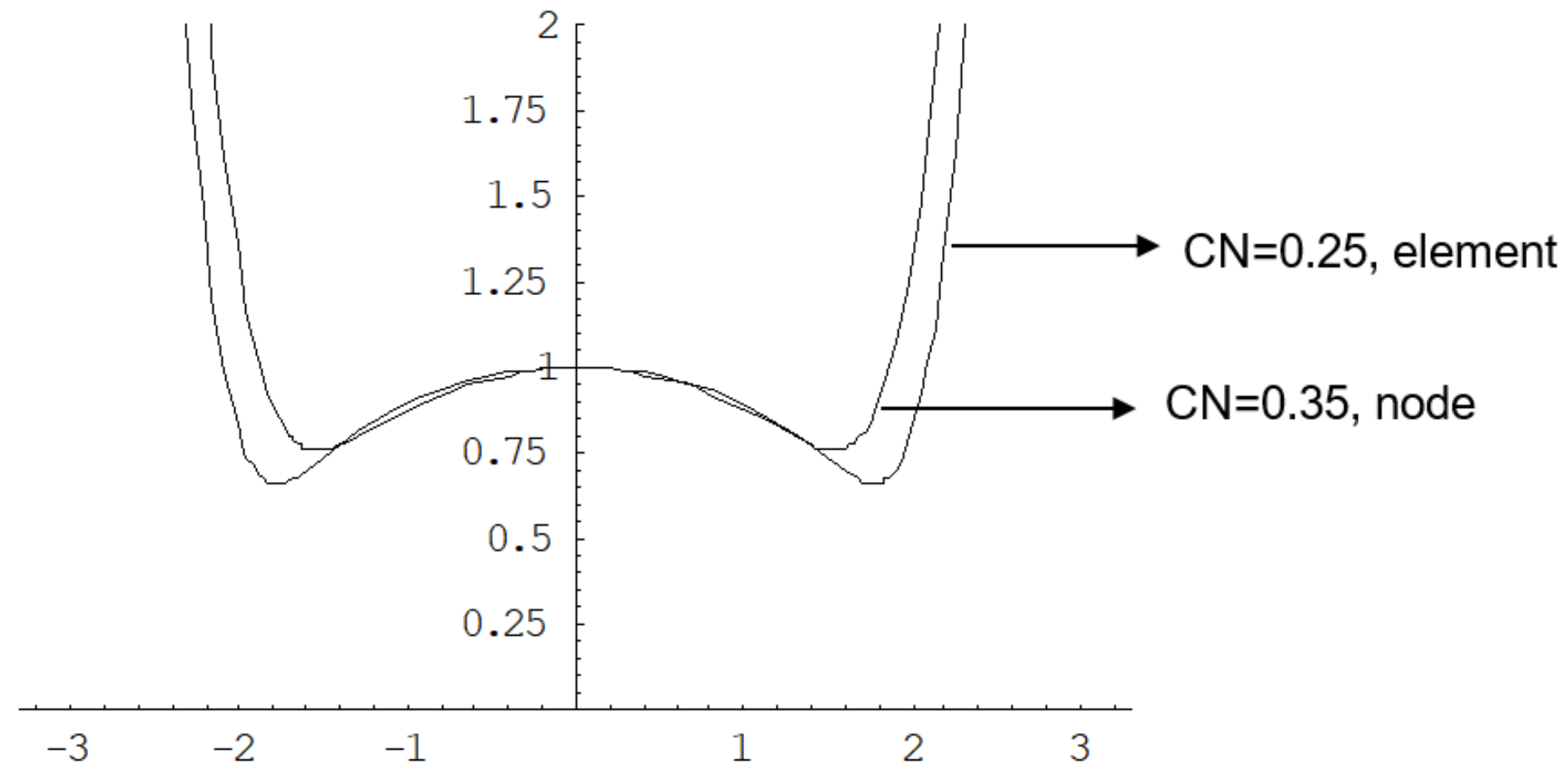

\section{Figure 1}

Explicit finite element scheme and Consistent nodal solution 


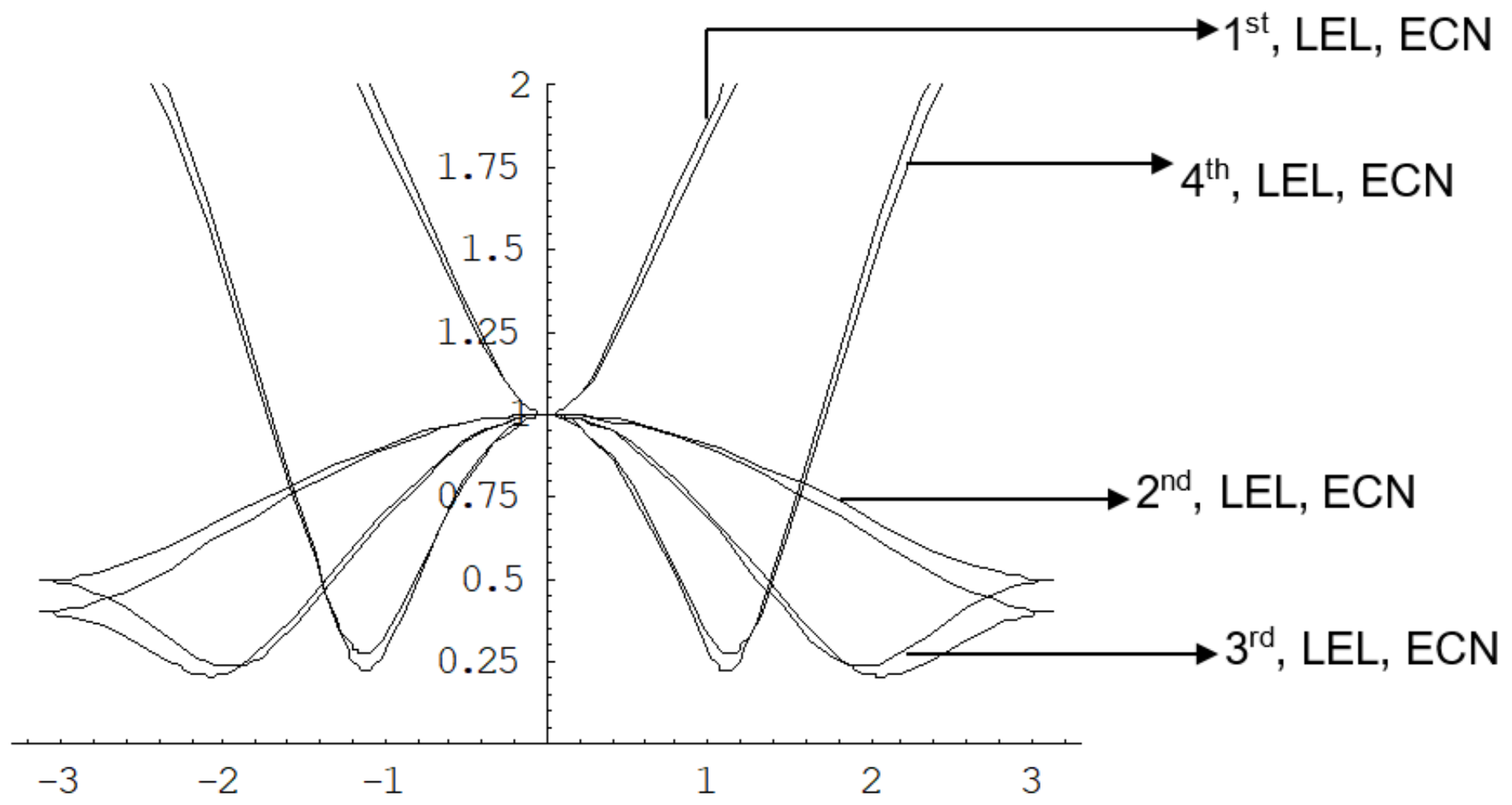

Figure 2

Lower bound nodal solution $(\mathrm{CN}=3 / 4)$ and Eigen-value solution $(\mathrm{CN}=0.70)$

(LEL = lumped, explicit, lower bound, ECN = eigen-value, consistent, nodal)

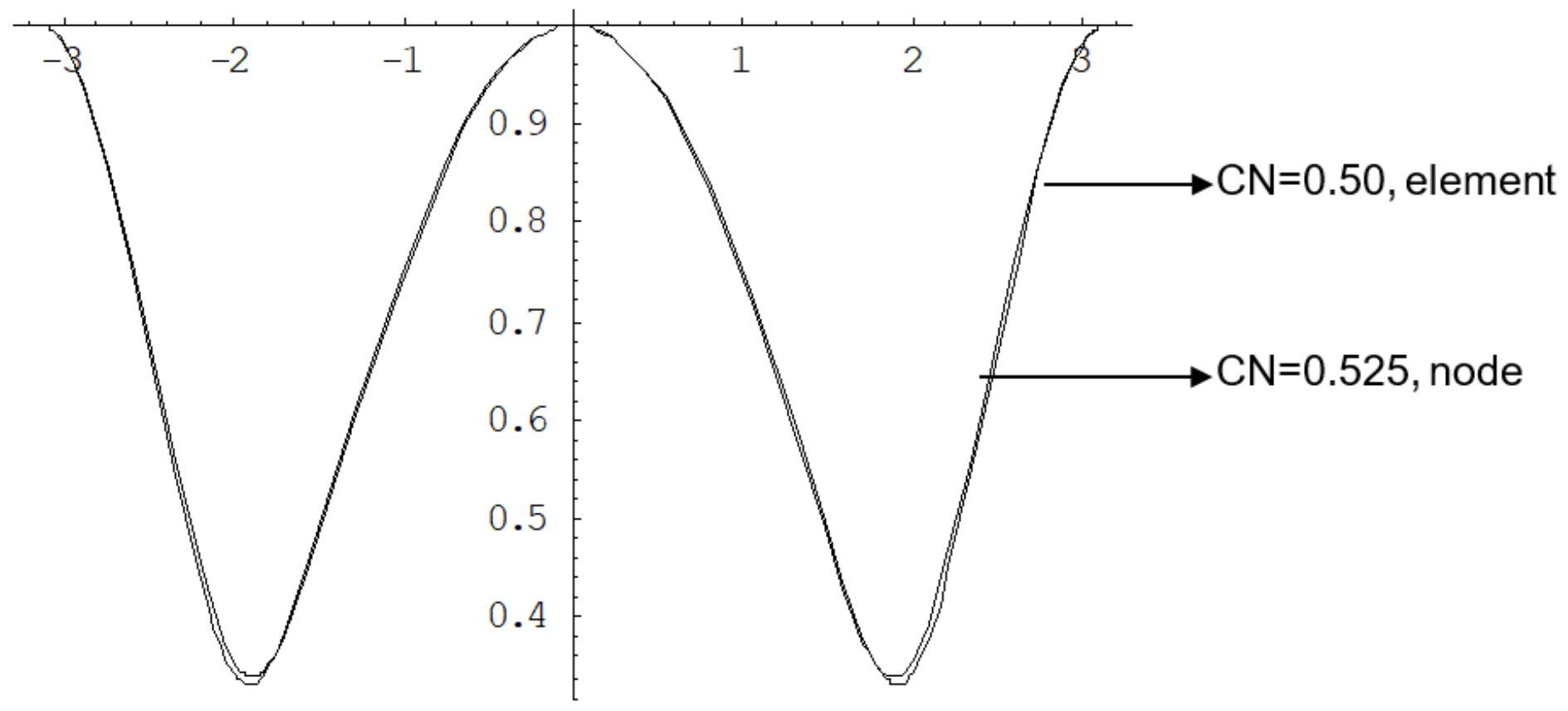

Figure 3 


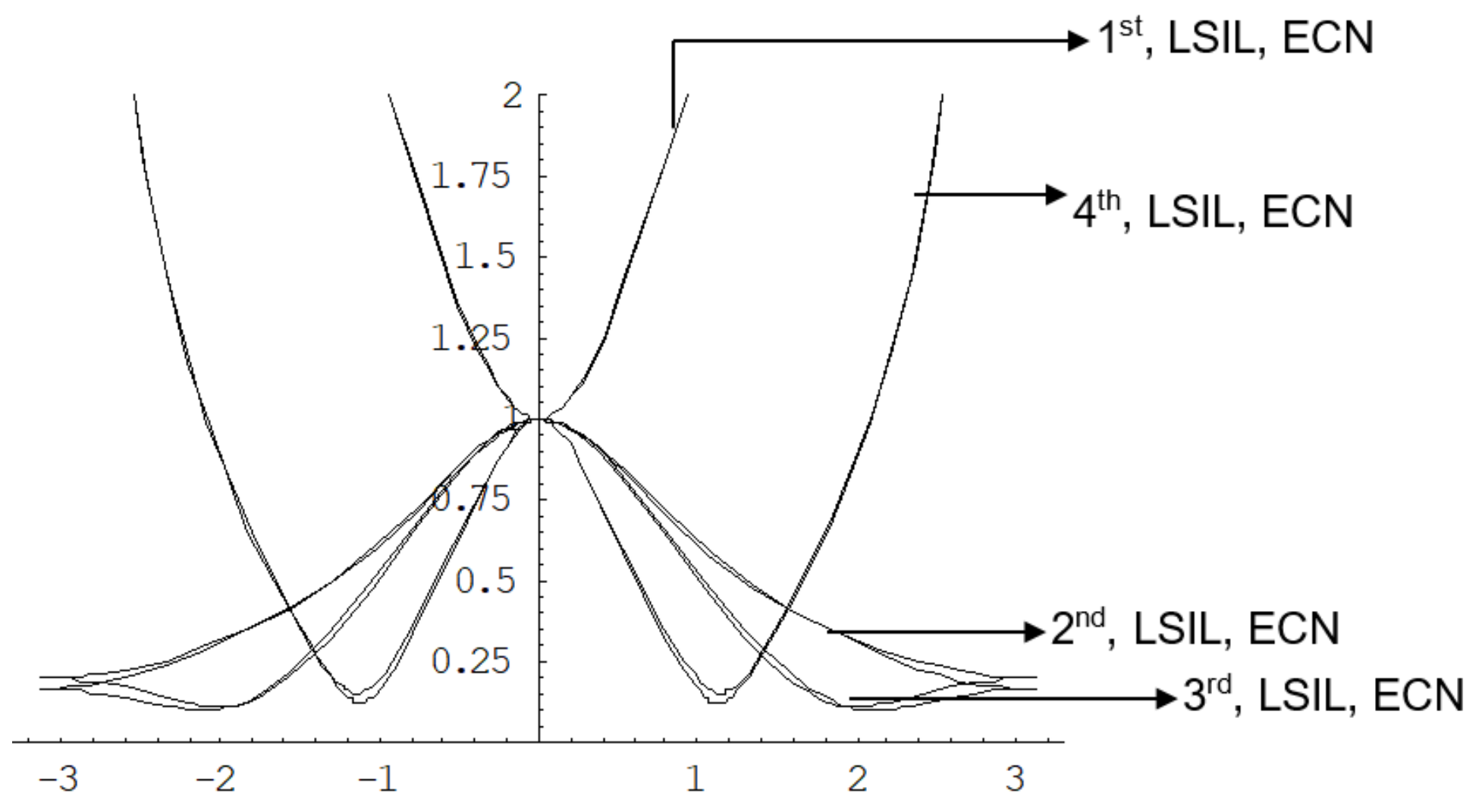

Figure 4

Lower bound nodal solution ( $\mathrm{CN}=3 / 2)$ and Eigen-value solution $(\mathrm{CN}=1.40)$

(LSIL = lumped, semi-implicit, lower bound, ECN = eigen-value, consistent, nodal) 


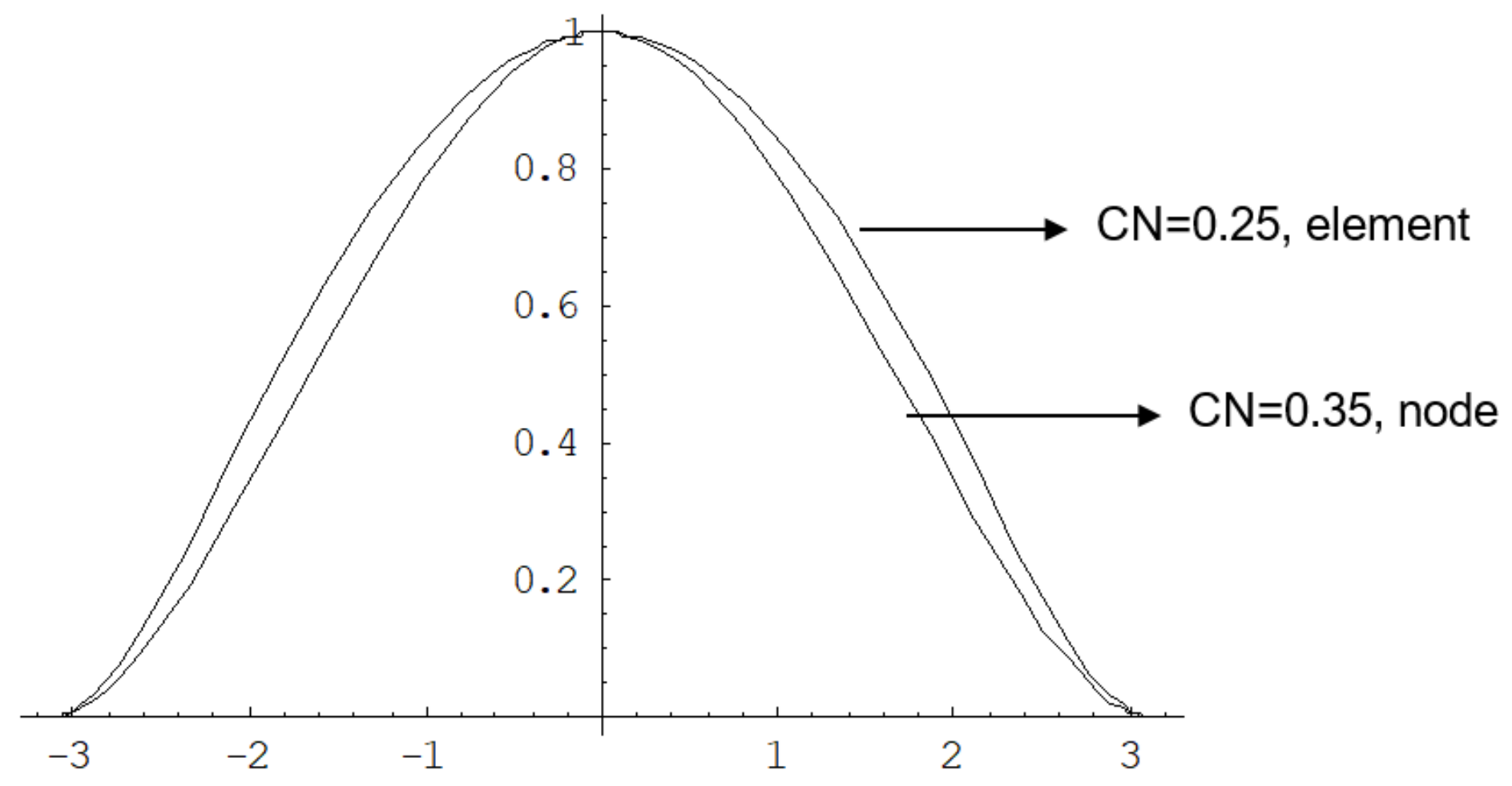

Figure 5

Implicit finite element scheme and Consistent nodal solution

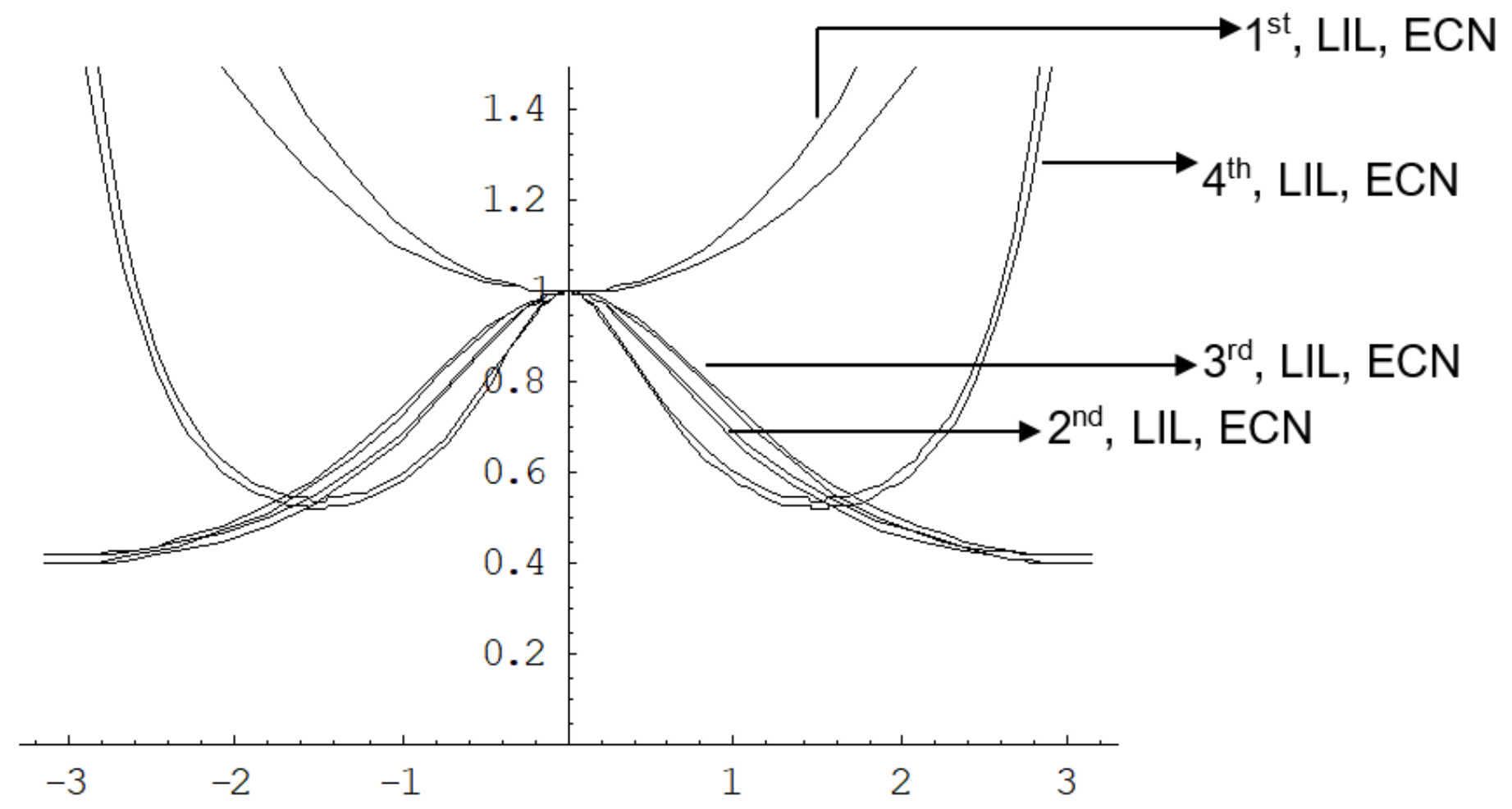


Figure 6

Lower bound nodal solution $(\mathrm{CN}=3 / 4)$ and Eigen-value solution $(\mathrm{CN}=0.70)$

$(\mathrm{LIL}=$ lumped, implicit, lower bound, ECN = eigen-value, consistent, nodal) 